[Frontiers in Bioscience 9, 192-200, January 1, 2004]

\title{
GRB7 IN INTRACELLULAR SIGNALING AND ITS ROLE IN CELL REGULATION
}

\author{
Tang-Long Shen and Jun-Lin Guan
}

Department of Molecular Medicine, Cornell University, Ithaca, New York 14853

\section{TABLE OF CONTENTS}

\author{
1. Abstract \\ 2. Introduction \\ 3. Structure \\ 3.1. Proline-rich region \\ 3.2. GM region \\ 3.2.1. PH domain \\ 3.2.2. RA domain \\ 3.2.3. BPS/PIR region \\ 3.3. $\mathrm{SH} 2$ domain \\ 4. Potential regulatory mechanisms \\ 4.1. Phosphorylation \\ 4.2. Localization \\ 4.3. Di/Oligomerization \\ 5. Grb7-mediated signaling \\ 5.1. FAK \\ 5.2. EphB1 receptor \\ 5.3 Phosphatidylinositol phosphates \\ 6. Cellular functions and physiological relevance of Grb7 \\ 6.1. Mitogenesis \\ 6.2. Cell migration \\ 7. Perspective \\ 6.3. Cancers \\ 8. Acknowledgments \\ 9. References
}

\section{ABSTRACT}

Grb7 is an adaptor molecule that mediates signal transduction from multiple cell surface receptors to various downstream signaling pathways. Grb7 and its related family member Grb10 and Grb14 share a conserved molecular architecture including an amino-terminal proline-rich region, a central segment termed the GM region (for Grb and Mig) which includes a PH domain and shares sequence homology with the Caenorhabditis elegans protein, Mig-10, and a carboxyl-terminal $\mathrm{SH} 2$ domain. Grb7/10/14 family proteins are phosphorylated on serine/threonine as well as tyrosine residues, although the functional significance of such phosphorylation is incompletely understood. Grb7/10/14 family proteins are mainly localized in the cytoplasm, but have been observed at the plasma membrane, focal contacts, or mitochondria under certain conditions. A large number of receptor tyrosine kinases and other signaling molecules can associate with Grb7/10/14 family proteins, mostly through the SH2 domains, although the functional consequences of such interactions have not been well characterized in most cases. Recent studies have suggested that various isoforms of Grb10 play important roles in mediating insulin/insulinlike growth factor regulation of cell proliferation and apoptosis, whereas Grb7 mediates signaling pathways from
FAK and EphB1 receptor to regulate cell migration, which is also implicated in tumor progression. This review will discuss the current understanding of Grb7 mediated signal transduction pathways and their role in the regulation of various cellular functions.

\section{INTRODUCTION}

Grb7 encodes a 535-amino acid protein, which is composed of an amino-terminal proline-rich region, a central segment termed the GM region (for Grb and Mig) with a pleckstrin homology $(\mathrm{PH})$ domain, and a carboxylterminal src-homology 2 (SH2) domain, all of which are involved in protein-protein interaction or protein-lipid interaction (1). Grb7 is an adaptor molecule and lacks intrinsic enzymatic activity. It functions to couple signals from cell surface receptors to specific downstream pathways inside the cell. Grb7 shares significant sequence homology with Grb10 and Grb14, which together form the Grb7/10/14 family adaptor proteins. In addition, the Caenorhabtidis elegans mig10 gene represents a conservation of this gene family among different species. According to newly adopted nomenclature (2), at present, there are two splice variants of Grb7 (hGrb7 and hGrb7V), 


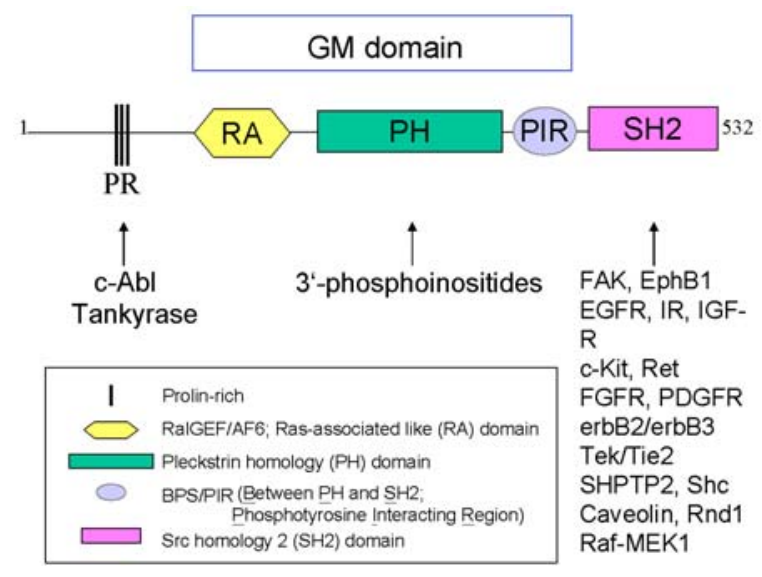

Figure 1. Domain scheme of the Grb7 family proteins. Grb7 protein family is composed of Grb7, Grb10 and Grb14. These proteins are homologous with Mig10 protein from C. elegan. Numerous molecules have been found to associate with $\mathrm{Grb} 7$ through distinct domains/motifs as shown here. PR, proline-rich; RA, RalGEF/AF6 or Rasassociated like; PH, Pleckstrin homology; PIR (BPS), phosphotyrosine interacting region (between $\mathrm{PH}$ and $\mathrm{SH} 2$ ); SH2, Src homology 2; GM, Grb and Mig.

six variants of Grb10 (mGrb10alpha, hGrb10beta, hGrb10gamma, mGrb10delta, hGrb10epsilon, and hGrb10zeta), and Grb14 variants such as hGrb14, mGrb14, and rGrb14 (2).

This family of proteins shares a highly conserved structure (Figure 1). However, they exhibit distinctive expression patterns among tissues as shown by measuring mRNA levels. For instance, in mouse, Grb7 is highly expressed in kidney and liver cells and at a lower level in ovaries, testes and lung tissues $(3,4)$. All of these tissues express very little of $\operatorname{Grb} 10(5,6)$. In humans, all members of the Grb7/10/14 family are abundantly expressed in pancreatic tissues $(1,7,8)$, yet their amount is varied in kidney and liver (7-9). In addition, the expression of Grb7 is correlated with kidney development in mice. In comparison, Grb10 is expressed in rat liver tissues during embryogenesis but becomes undetectable shortly after birth $(10,11)$. These tissue-specific distributions may account for their diverse roles and responses to extracellular signaling proteins (see below). In this section, we will discuss the functional structure, potential regulatory mechanisms, signaling, and biological role of Grb7.

\section{STRUCTURE}

\subsection{Proline-rich region}

Several minimal consensus repeats, PXXP, are located within the amino-terminal region of Grb7/10/14 family proteins. These consensus sequences can modulate protein-protein interactions by binding to the src-homology 3 (SH3) domain of various proteins (12). However, no proteins have yet been identified that interacts with this proline-rich region of Grb7. In contrast, in vitro experiments reveal that the $\mathrm{SH} 3$ domain of c-Abl may bind to the proline-rich region of Grb10, yet its biological function is unclear (7). Recently, the ankyrin repeats in human Tankyrase 2, a poly-ADP-ribose polymerase, has been reported to associate with the N-terminal 110 amino acids of Grb14 (13).

\subsection{GM region}

This approximately 300 amino acids sequence is named for high sequence homology (approximately 50\% amino acid identity) between the Grb7/10/14 proteins and Mig-10 from C. elegans (14). This GM region includes a central pleckstrin homology $(\mathrm{PH})$ domain, and is flanked by a putative RA (Ral GDS/AF6 or Ras-Associating) domain at the amino-terminus and a functional region of about 50 amino acids termed BPS (between $\mathrm{PH}$ and $\mathrm{SH} 2$ domains) or PIR (phosphotyrosine interacting region) at the carboxyl terminus $(9,14-16)$.

\subsubsection{PH domain}

By amino acid sequence alignment analysis, Grb7/10/14 family proteins bear a homologous domain called pleckstrin homology (PH) domains (9). $\mathrm{PH}$ domains have secondary and tertiary module conservation, spanning about 110 amino acids. Here, seven $\beta$ strands form a sandwich that is capped on one end of one $\alpha$ helix (17). Almost all the known PH domains bind to phospholipids, varying in affinity and specificity. The interaction between phospholipids and $\mathrm{PH}$ domains may govern many cellular signaling events such as membrane localization, conformational changes, vesicle trafficking, and cytoskeletal organization (18). Other functions of $\mathrm{PH}$ domains are to act as protein-protein and/or protein-lipid interaction modules $(19,20)$.

Recent data from our laboratory confirm that the PH domain of Grb7 can bind to specific phosphoinositides. This interaction between Grb7 and phosphoinositides requires the conserved critical residues found in other $\mathrm{PH}$ domains during protein-phosphoinositides binding, thus modulating the Grb7-mediated signaling events (16) (see below).

\subsection{2. $R$ A domain}

The presence of the RA domain in the Grb7/10/14 super family was proposed according to sequence homology analysis (15). This conserved Ras superfamily binding domain (RBD) has been characterized in a variety of proteins, several of which are known to be RasGTPase effectors such as RalGDS (Ral guanine nucleotide dissociation stimulator or called RalGEF), afadin/AF-6 and c-Raf (21). This suggests the interesting possibility that Grb7/10/14 family members might serve as effectors and regulate the Ras signaling pathways that are important for cell proliferation. However, several different approaches failed to detect the interaction between Grb7/10/14 family proteins and small GTPases (10). Yet, the significance of the Grb7/10/14 RA domain remains unclear.

\subsubsection{BPS/PIR region}

A novel functional region involved in regulating signaling in the Grb7/10/14 family exists between the PH and $\mathrm{SH} 2$ domain. This region participates in $\mathrm{SH} 2$ domain dependent and independent interactions (4, 24, 25). 
However, it is not certain if this region forms a folded unit like the SH2 domain. The mechanism that influences the binding specificity and affinity of the BPS/PIR and SH2 domains needs to be elucidated (also see below).

\subsection{SH2 domain}

The SH2 domain is an approximately 100 amino acid-long protein module that folds into a hydrophobic phosphotyrosine-binding core. In addition to the conserved FLVRES motif, three positively charged residues and several residues on the phosphotyrosine binding loop provide stable hydrogen bonding to phosphotyrosine (see reviews in $(26,27))$. The binding of $\mathrm{SH} 2$ domains to phosphotyrosine motifs recruit their host proteins to activated upstream targets (e.g. tyrosine kinase receptors), and mediates signaling events in a spatial and temporal manner. The binding selectivity of the $\mathrm{SH} 2$ domains to the phosphotyrosine motifs depends on the sequence context around it, particularly the +1 and +3 positions near the $C$ terminus of the phosphotyrosine (28).

High amino acid sequence homology (approximately $70 \%$ amino acid identity) exists among the $\mathrm{SH} 2$ domains of the Grb7/10/14 family members (1). However, each member of the Grb7/10/14 family proteins seems to have different preferences for binding partners. For instance, the Grb7 SH2 domain binds to erbB2; but the Grb14 SH2 domain cannot associate with this protein (29). Similarly, hGrb7 associates with FAK through its SH2 domain (30), whereas hGrb10gamma does not bind to FAK (7). These results suggest that each Grb7/10/14 family member has evolved to interact with its specific functional partners. Furthermore, these interactions may bring different outcomes in cell signaling. For example, the SH2 domain deleted form of Grb7, Grb7V, is reported to be associated with invasive esophageal carcinomas (31). Compared to wild type Grb7, this isoform does not respond to EGF treatment, presumably due to its lack of binding to EGF receptors. Analysis of the binding motifs of these binding partners suggests that Grb7/10/14 family proteins association with other proteins is highly promiscuous. Nonetheless, using phage display screening, a motif containing the Tyr-X-Asn (YXN) was identified and was found to specifically bind to the $\mathrm{SH} 2$ domain of $\mathrm{Grb} 7$ but not the SH2 domain of Grb2 and Grb14 (32). Moreover, synthetic Grb7-binding peptides were able to specifically inhibit the association of Grb7 with the ErbB family of RTKs in a dose-dependent manner. These studies highlight the functional importance of the SH2 domain in Grb7/10/14 family proteins.

\section{POTENTIAL REGULATORY MECHANISMS}

\subsection{Phosphorylation}

Serine/threonine phosphorylation is constitutive in the Grb7/10/14 family proteins. For example, in the quiescent state, Grb7 is phosphorylated on serine and threonine residues, and treatment with growth factors (EGF or heregulin) does not alter its phosphorylation status (33, 34). In contrast to Grb7, mGrb10 and hGrb10gamma have been reported to be serine phosphorylated upon EGF and insulin treatment, respectively $(9,35)$. Other evidence suggests that the MAPK kinase (MEK) might serve as a potential hGrb10gamma kinase particularly during insulin signaling (35). Similarly, Grb14 is also phosphorylated on serine or threonine residues, yet its phosphorylation is stimulated by PDGF and not EGF (8) or FGF-2 treatment (36). Recently, PKCzeta has been reported to phosphorylate Grb14 upon insulin stimulation, thereby resulting in the inhibition of insulin signaling (37). However, the functional significance of serine and/or threonine phosphorylation of the Grb7/10/14 family members is not fully understood.

Recently, several laboratories have observed tyrosine phosphorylation of Grb7 (38-40). Jones and his colleagues first showed that Grb7 binds to Tyr-1100 of the Tek receptor tyrosine kinase and mutation of this residue to Phe abolishes both Grb7 binding and its tyrosine phosphorylation by Tek (38). Similarly, tyrosine phosphorylation of Grb7 was detected when FAK was activated by replating cells on fibronectin, or when FAK was overexpressed in CHO cells (41). Furthermore, Grb7 phosphorylation is stimulated only by wild-type FAK and not by kinase-defective FAK, or by the FAK mutant (Y397 F) which lacks the binding site for Grb7. In addition, overexpression of FAK increases tyrosine phosphorylation of Grb7 in SYF cells (deficient in Src, Yes and Fyn expression), suggesting that the FAK-dependent Grb7 phosphorylation does not require Src family tyrosine kinases. Furthermore, treatment of cells with PI-3 kinase inhibitors, LY294002 and wortmannin, inhibits the tyrosine phosphorylation of Grb7 by FAK, suggesting the requirement of PI-3 kinase activity in the activation of Grb7 (16). In contrast to Grb7, Grb10 was found to be tyrosine phosphorylated by Src and Fyn in insulin treated CHO-IR cells (42). A Tyr-67 to Gly mutation of Grb10 abolished its tyrosine phosphorylation by Src/Fyn even though this mutant showed increased binding to IR. In a recent report, Grb10 is also shown to be tyrosine phosphorylated in response to VEGF and this phosphorylation is partly mediated by Src, although the kinase activity of VEGF receptor also contributes to the phosphorylation (43). Tanaka and his colleagues also reported tyrosine phosphorylation of Grb7 in human esophageal carcinoma cells when treated with EGF or when replated on fibronectin-coated dishes (40). Moreover, the tyrosine phosphorylation of Grb7 by EGF treatment is independent of integrin activation, thereby suggesting that Grb7 is a substrate for both FAK and the EGF receptors. Recently, we have identified an interaction between Grb7 and the EphB1 receptor. The tyrosine phosphorylation of Grb7 can be stimulated by EphB1 activation, but not its kinase-deficient mutant (44). There are several tyrosine residues within Grb7, and the determination and characterization of the tyrosine phosphorylation sites on Grb7 will help to unravel the signal transmission mechanisms of the EphB1/Grb7 complex.

\subsection{Localization}

Appropriate adaptor protein localization results in a concert of upstream signaling activation and recruitment of downstream effectors. In fact, hGrb7 can be detected in both the cytoplasm and focal adhesions $(16,41)$. Deletion of the $\mathrm{SH} 2$ domain of Grb7 abolishes the localization at 


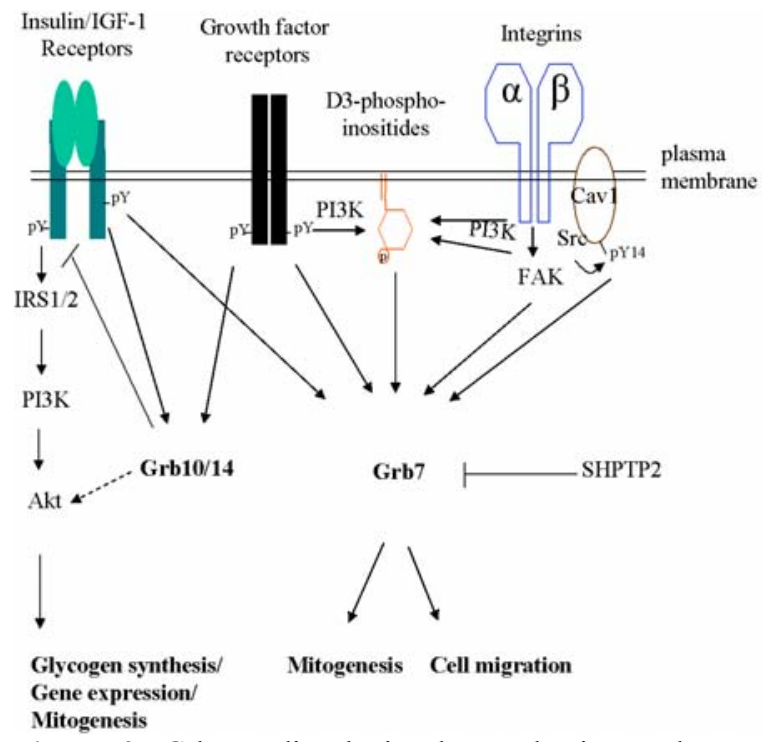

Figure 2. Grb7-mediated signal transduction pathways. Grb7 family proteins are cytosolic adaptor molecules that couple signals from the cell surface to various intracellular signaling cascades. They function as either positive or negative regulators of different receptors and regulate several cellular functions such as cell migration, mitogenesis and gene expression. Some of wellcharacterized Grb7-mediated signaling pathways are shown. See the text for more detail.

focal contacts and impairs its stimulation of cell migration, suggesting that the SH2 domain is critical for Grb7 to localize at the focal contact sites and its role in mediating cell migration (41). In addition, the $\mathrm{PH}$ domain interaction with phospholipids may also contribute to Grb7's localization by targeting it to the plasma membrane. In fact, expression of the Grb7 PH domain has shown similar plasma membrane distribution as other $\mathrm{PH}$ domain containing proteins (16), thus facilitating Grb7/FAK interaction within focal contacts. However, studies from our laboratory suggest that the $\mathrm{PH}$ domain of Grb7 is not required for its localization within focal adhesion but rather is involved in regulating its conformation (see below).

\subsection{Di/Oligomerization}

Dimerization or/and oligomerization has become an emerging and universal phenomenon in order to modulate receptor integrity and function (e.g. G proteincoupled receptors, EGF receptors), as well as specificity and activity of many intracellular signaling molecules (e.g. p53, JAK-STAT) (see reviews in (47-49)). For example, the dimerization/oligomerization of EGF receptors allows their kinase domains to be positioned next to each other, thereby facilitating cross-phosphorylation, activation, substrate phosphorylation and stability (49). Dimerization of the same or different activated STAT proteins (hetero- or homo-dimer) can also transduce a distinctive downstream signal and regulate gene expression (50). There are also reports suggesting that the Grb7/10/14 family members may dimerize to form homo- or hetero- dimers/oligomers. Grb10 is shown to dimerize/oligomerize through interactions between the N-terminal domain of one molecule and the PH/BPS/SH2 domains of another (51). In addition, Grb10 is reported to bind Grb7 in yeast twohybrid assays (unpublished data by J. Cooper in (51)). However, it remains to be evaluated how dimerization/oligomerization affects Grb7/10/14's activity and signaling.

\section{GRB7-MEDIATED SIGNALING}

Originally, the Grb7/10/14 family proteins were cloned based on their interactions with the EGF receptor, using the CORT (cloning of receptor target) system (3). Other tyrosine kinase receptors, such as HER2/erbB2, Ret, PDGFR, erbB3 receptor, Tek/Tie2, c-Kit/SCFR, IR, IGF-1 receptor, FGF receptor, HGF receptor, KDR/VEGF receptor, and EphB1 also have been demonstrated as binding partners for the Grb7/10/14 family proteins. In addition, the Grb7/10/14 family proteins are able to interact with other families of proteins, for example, protein kinases (e.g. Jak2, FAK, Raf1, MEK1, Bcr-Abl, Tec kinase, Akt), phosphatase (e.g. SHPTP2), adaptor protein (e.g. Shc, ZIP), small GTPases (e.g. Rnd1), ubiquitin ligases (e.g. Nedd4) and caveolin-1 (1). Most of these interactions are through the interaction of the Grb7/10/14 SH2 domain with phosphotyrosine motifs in the binding partners in a signal dependent manner. Grb7 also favors association with IR rather than EGFR, FGFR and Ret receptors (4). As mentioned earlier, high expression of Grb7 in mouse liver may be a good indication of the physiological importance of Grb7 in insulin signaling. Yet, the current evidence does not provide any data for the binding of Grb7 to IR and its role in the promotion of IR signaling. In the following section, we will discuss some of the best-characterized Grb7-mediated signaling pathways, which are summarized in Figure 2.

\subsection{FAK}

FAK localizes at focal adhesions upon the activation of integrins and has been implicated to play a key role in integrin-mediated signal transduction during cell migration (64-67). The Tyr-397 of FAK is a major autophosphorylation site in vitro and in vivo. This phosphotyrosine 397 acts as a docking site for several SH2 domain-containing signal molecules including Src, PI-3 kinase, and PLCgamma-1, all of which play roles in signaling pathways during cell migration (68-71). Grb7 also has been reported to interact with the Tyr-397 of FAK through its $\mathrm{SH} 2$ domain in an adhesion-dependent manner (30). Interestingly, no competition of binding to the FAK Tyr-397 between Grb7 and Src is observed (30) in agreement with the differential binding capacity of this motif to different $\mathrm{SH} 2$ domains in a spatial or/and temporal fashion. Indeed, a FAK mutant, D395A, selectively disrupts FAK binding to Grb7, but allows FAK association with Src (72). On the other hand, the recruitment of Grb7 through its $\mathrm{SH} 2$ domain by FAK can facilitate its signaling and cellular functions cooperatively with other signaling proteins (e.g. PI-3 kinase) at focal adhesions and/or the plasma membrane. For example, evidence from our laboratory has indicated that the PH domain of Grb7 serves as a downstream target of PI-3 kinase activity, which is demonstrated by the association of Grb7's PH domain with 
D-3 phosphoinositides, the products of PI-3 kinase, in an integrin-dependent manner (16). Furthermore, this association is necessary for Grb7 phosphorylation by FAK and that is required for the stimulation of cell migration by Grb7 $(16,41)$. In support of this, a chimeric Grb7 protein was constructed by replacing the SH2 domain with FAT sequence (Focal Addhesion Targeting sequence). This FAT Grb7-FAT chimera was constitutively targeted to the focal contact sites and showed significant associations with phospholipids, is tyrosine phosphorylation by FAK and stimulated cell migration when compared to the $\mathrm{SH} 2$ domain truncation mutant of Grb7 $(16,41)$. Lastly, Grb7 has been shown as a substrate for FAK but not Src family kinases (41). This event couples the signal from FAK to downstream effectors, thus regulating cell migration. Identification of the potential mediator(s), the SH2 domaincontaining protein(s), may shed light toward the understanding of FAK/Grb7-mediated signal transduction during cell migration.

\subsection{EphB1 receptor}

The Eph family of receptor tyrosine kinases is comprised of two subfamilies: first, the EphA kinases which bind to ephrinA ligands and are anchored to cytoplasmic membrane through a glycosyl phosphatidylinositol linkage and, the other EphB kinases which bind to ephrinB ligands and have a single transmembrane domain. The Eph-ephrin interaction and resultant bi-directional signaling have been implicated in diverse biological processes including nervous system development, angiogenesis, and neural synapse formation and maturation $(73,74)$. In two independent yeast twohybrid screenings, Grb10 was identified as an interacting protein by using the cytoplasmic domain of EphB1 as "bait"; similarly, using Grb7 as "bait", Grb7 was also shown to interact with the cytoplasmic domain of EphB1 $(44,75)$. Interestingly, the interaction of Grb7 with the EphB1 receptor occurs in an autophosphorylation dependent manner and is enhanced by ligand-activation. The Tyr-928 of EphB1 receptor was demonstrated to be the major binding site for both Grb7 and Grb10 (44, 75). A potential role of the Grb7/EphB1 complex was demonstrated during cell migration where co-expression of Grb7 and EphB1 led to a synergistic effect. In addition, the $\mathrm{SH} 2$ domain of Grb7 was sufficient enough to abolish EphB1 induced migration (44). In contrast, there is no direct evidence presenting the effect of Grb10 in cell migration to date. However, Grb10 may also play a negative role in Eph receptor-mediated cell migration since it has been implicated to play an inhibitory role in other signaling pathways.

\subsection{Phosphatidylinositol phosphates}

The Grb7/10/14 family proteins may act as phosphoinositides-binding modules through their conserved PH domains. This implies that the Grb7/10/14-mediated signaling may involve the well-known secondary messengers: phosphatidylinositol (PtdIns) phosphates such as PtdIns 3,4- $\mathrm{P}_{2}$, PtdIns 3,5- $\mathrm{P}_{2}$, PtdIns 4,5- $\mathrm{P}_{2}$, and PtdIns $3,4,5-\mathrm{P}_{3}(18)$. Even though the $\mathrm{PH}$ domain is dispensable for recruitment, other functions may result through the recognition of specific phospholipids by the $\mathrm{PH}$ domain, causing Grb7/10/14 to modulate its conformation. In support of this, as discussed above, the binding of the Grb7's PH domain to phospholipids, i.e. D3- and/or D5phosphoinositides, is necessary for its phosphorylation by FAK (16). In addition, the phosphorylation of Grb10 by MEK in response to insulin is sensitive to the PI-3 kinase specific inhibitor, wortmannin, implying that Grb10's PH domain also requires the product(s) of PI 3-kinase for its activity (35). Further investigations of the PH domains of Grb7/10/14 family proteins are needed, as well as their phospholipid binding specificities and the resulting downstream signaling of these interactions.

\section{CELLULAR FUNCTIONS AND PHYSIOLOGICAL RELEVANCE OF GRB7}

\subsection{Mitogenesis}

All Grb7/10/14 family members interact with growth factor receptors in an activation dependent manner, suggesting that they might be involved in the regulation of cell proliferation. Several studies suggest a negative role of Grb10 in insulin and IGF-1 induced cell proliferation, through the inhibition of their downstream signaling, DNA synthesis, and cell growth, although contradicting results have been reported in other studies $(45,60)$. A number of studies also reveal that expression of exogenous Grb14 inhibits DNA synthesis in response to insulin, serum or FGF-2 $(25,36)$. The role for Grb7 in the regulation of cell proliferation is still unclear. Our laboratory has shown that overexpression of the Grb7's SH2 domain does not decrease cell cycle progression (72), although it inhibited cell migration under similar conditions (76). Conversely, a recent report has demonstrated that Grb7 is able to increase the anchorage-independent growth via binding to phosphotyrosine 14 of caveolin-1 (77).

\subsection{Cell migration}

As discussed earlier, the Grb7/10/14 family proteins share a high sequence homology with a Caenorhabditis elegans gene product, Mig-10 (9, 14, 34). The Mig-10 protein plays a role in long-range migration of neuronal cells during embryonic development (80). This sequence similarity raises the possibility that Grb7 or other family members may play a role in the regulation of migration of mammalian cells. In fact, correlation of Grb7 overexpression with increased invasion was also reported in esophageal carcinoma cells (40). Conversely, expression of an antisense Grb7 RNA lowers endogenous Grb7 protein levels and suppresses the invasive phenotype of these cells in vitro (31). Studies in our laboratory have confirmed the role of $\mathrm{Grb} 7$ in cell migration. First, inducible overexpression of Grb7 stimulates NIH3T3 cells to migrate toward the fibronectin in the Boyden chamber migration assay; whereas expression of its $\mathrm{SH} 2$ domain alone acts as a dominant negative mutant which reduces cell migration (30). Also, transient transfection of wild type Grb7 into $\mathrm{CHO}$ cells promotes cell motility on fibronectin, as shown by a time-lapse microscopy cell motility assay, whereas truncation mutants reduce motility (41). Thus, these results confirm that Grb7 plays a role in integrin-mediated cell migration. In addition, many growth factor receptors that have been known to interact with Grb7 also play a role in 
cell migration, thereby supporting a potential role for Grb7 in mediating cell migration. For instance, Lee et al. recently demonstrated that the binding of Grb7 to phosphotyrosine 14 (pY14) on caveolin-1 promotes EGF-stimulated cell migration (77). As mentioned earlier (see section 5.3.), Grb7 acts as a downstream transducer of the EphB1 receptor, and co-expression of Grb7 and EphB1 can synergistically enhance cell migration. Moreover, the SH2 domain of Grb7 can block the downstream signaling and thus reduces cell migration (44).

In contrast to Grb7, the other members, Grb10alpha, Grb10gamma and Grb14 show little effect on cell migration on fibronectin coated plates (41). Thus, this suggests that the latter may play different cellular functions such as cell proliferation (see above) rather than cell migration.

\subsection{Cancers}

Grb7 is co-amplified with the EGF or HER2/erbB2 receptors and is potentially involved in promoting metastasis and invasion of numerous breast tumors $(31,34$, 81,82 ). In addition, comparative genomic hybridization microarray analyses of chromosome $17 \mathrm{q}$, where Grb7 is located on the human genome, reveals an increase in copy number and/or expression levels of a minimal amplicon region, including ErbB2 and Grb7, in breast cancers (83), gastric cancers (84) and testicular germ cell tumorigenesis (85). Grb10 and Grb14 are also expressed in several breast or prostate cancer cell lines $(8,35)$. However, the coamplification of Grb7 and HER/erbB2 may be due to their close chromosomal/spatial linkage, thus Grb7's involvement may be a coincidence and not a requirement. After all, HER2/erbB2, a member of the EGF receptor family, is known to be amplified and overexpressed in $\sim 25 \%$ of human breast cancer (86). Yet, Grb7 is strongly suggested to be involved, as it can form a complex in vivo. This evidence implies that both Grb7 and HER2/ErbB2 are involved in the same pathway. This downstream signaling of Grb7 may be highly amplified in certain breast cancer cell lines. In addition, the results of Tanaka et al reinforces Grb7's role in cancer progression since coexpression of Grb7 with Her2 is related to esophageal carcinoma as well as extramucosal tumor invasion (82).

\section{PERSPECTIVE}

Numerous reports indicate that the activity of tyrosine kinases such as EGFR, FAK and EphB1 is capable of activating Grb7's downstream signaling pathways. For example, Grb7 is a substrate for FAK and its phosphorylation is independent of Src. Previous studies have suggested that phosphorylation of Grb7 by FAK is required for Grb7-stimulated cell migration. Similarly, the phosphorylation of Grb7 by EphB1 kinase can also promote cell migration on fibronectin. Thus, it will be interesting to determine the phosphotyrosine residue(s) on Grb7. This information will shed light on the identification of downstream signaling molecule(s) and pathway(s) in FAK- or EphB1-mediated cell migration.

The metabolic products of PI-3 kinase were demonstrated to bind the Grb7's PH domain and that was required for the Grb7's phosphorylation by FAK. Currently, the molecular mechanism of the Grb7's PH domain binding to phosphoinositides and why this is important for its accessibility to FAK is not understood. Crystal structure of Grb7 complexes with specific inositides will provide a physical resolution to understand this. Nevertheless, the evidence in our studies has demonstrated an example of a cooperative mechanism of FAK interacting proteins in FAK-mediated signaling. Further studies should be carried out, for example, a real-time study, to examine other potentially cooperative events in regulating FAK signaling in a spatial and/or temporal manner.

\section{ACKNOWLEDGMENTS}

This work was supported by NIH grant GM48050 to J.-L. Guan. We thank Lee Ann Cooper, Dan Rhoads, Hans Salamanca-Granados and Patrick Sturges for critical reading of the manuscript.

\section{REFERENCES}

1. Han, D. C., Shen, T. L., and Guan, J. L. The Grb7 family proteins: structure, interactions with other signaling molecules and potential cellular functions. Oncogene 20, 6315-6321 (2001)

2. Daly, R. J. The Grb7 family of signalling proteins. Cell Signal 10, 613-618 (1998)

3. Margolis, B., Hu, P., Katzav, S., Li, W., Oliver, J. M., Ullrich, A., Weiss, A., and Schlessinger, J. Tyrosine phosphorylation of vav proto-oncogene product containing $\mathrm{SH} 2$ domain and transcription factor motifs. Nature 356, 71-74 (1992)

4. Kasus-Jacobi, A., Bereziat, V., Perdereau, D., Girard, J., and Burnol, A. F. Evidence for an interaction between the insulin receptor and Grb7. A role for two of its binding domains, PIR and SH2. Oncogene 19, 2052-2059 (2000)

5. Liu, F., and Roth, R. A. Grb-IR: a SH2-domaincontaining protein that binds to the insulin receptor and inhibits its function. Proc Natl Acad Sci U S A 92, 1028710291 (1995)

6. O'Neill, T. J., Rose, D. W., Pillay, T. S., Hotta, K., Olefsky, J. M., and Gustafson, T. A. Interaction of a GRBIR splice variant (a human GRB10 homolog) with the insulin and insulin-like growth factor I receptors. Evidence for a role in mitogenic signaling. J Biol Chem 271, 2250622513 (1996)

7. Frantz, J. D., Giorgetti-Peraldi, S., Ottinger, E. A., and Shoelson, S. E. Human GRB-IRbeta/GRB10. Splice variants of an insulin and growth factor receptor-binding protein with $\mathrm{PH}$ and SH2 domains. J Biol Chem 272, 26592667 (1997)

8. Daly, R. J., Sanderson, G. M., Janes, P. W., and Sutherland, R. L. Cloning and characterization of GRB14, a novel member of the GRB7 gene family. J Biol Chem 271, 12502-12510 (1996)

9. Ooi, J., Yajnik, V., Immanuel, D., Gordon, M., Moskow, J. J., Buchberg, A. M., and Margolis, B. The cloning of Grb10 reveals a new family of $\mathrm{SH} 2$ domain proteins. Oncogene 10, 1621-1630 (1995)

10. Leavey, S. F., Arend, L. J., Dare, H., Dressler, G. R., Briggs, J. P., and Margolis, B. L. Expression of Grb7 growth factor receptor signaling protein in kidney 
development and in adult kidney. Am J Physiol 275, F770776 (1998)

11. Gruppuso, P. A., Boylan, J. M., and Vaslet, C. A. Identification of candidate growth-regulating genes that are overexpressed in late gestation fetal liver in the rat. Biochim Biophys Acta 1494, 242-247 (2000)

12. Kay, B. K., Williamson, M. P., and Sudol, M. The importance of being proline: the interaction of proline-rich motifs in signaling proteins with their cognate domains. Faseb J 14, 231-241 (2000)

13. Lyons, R. J., Deane, R., Lynch, D. K., Ye, Z. S., Sanderson, G. M., Eyre, H. J., Sutherland, G. R., and Daly, R. J. Identification of a novel human tankyrase through its interaction with the adaptor protein Grb14. J Biol Chem 276, 17172-17180 (2001)

14. Manser, J., Roonprapunt, C., and Margolis, B. C. elegans cell migration gene mig-10 shares similarities with a family of $\mathrm{SH} 2$ domain proteins and acts cell nonautonomously in excretory canal development. Dev Biol 184, 150-164 (1997)

15. Wojcik, J., Girault, J. A., Labesse, G., Chomilier, J., Mornon, J. P., and Callebaut, I. Sequence analysis identifies a ras-associating (RA)-like domain in the $\mathrm{N}$ termini of band 4.1/JEF domains and in the Grb7/10/14 adapter family. Biochem Biophys Res Commun 259, 113120 (1999)

16. Shen, T. L., Han, D. C., and Guan, J. L. Association of Grb7 with phosphoinositides and its role in the regulation of cell migration. J Biol Chem 277, 29069-29077 (2002)

17. Lemmon, M. A., and Ferguson, K. M. Signal-dependent membrane targeting by pleckstrin homology $(\mathrm{PH})$ domains. Biochem J 350 Pt 1, 1-18 (2000)

18. Lemmon, M. A., Ferguson, K. M., and Abrams, C. S. Pleckstrin homology domains and the cytoskeleton. FEBS Lett 513, 71-76 (2002)

19. Gibson, T. J., Hyvonen, M., Musacchio, A., Saraste, M., and Birney, E. PH domain: the first anniversary. Trends Biochem Sci 19, 349-353 (1994)

20. Tsukada, S., Simon, M. I., Witte, O. N., and Katz, A. Binding of beta gamma subunits of heterotrimeric $G$ proteins to the $\mathrm{PH}$ domain of Bruton tyrosine kinase. Proc Natl Acad Sci U S A 91, 11256-11260 (1994)

21. Ponting, C. P., and Benjamin, D. R. A novel family of Ras-binding domains. Trends Biochem Sci 21, 422-425 (1996)

22. Geyer, M., Herrmann, C., Wohlgemuth, S., Wittinghofer, A., and Kalbitzer, H. R. Structure of the Rasbinding domain of RalGEF and implications for Ras binding and signalling. Nat Struct Biol 4, 694-699 (1997)

23. Nassar, N., Horn, G., Herrmann, C., Scherer, A., McCormick, F., and Wittinghofer, A. The 2.2 A crystal structure of the Ras-binding domain of the serine/threonine kinase c-Raf1 in complex with Rap1A and a GTP analogue. Nature 375, 554-560 (1995)

24. He, W., Rose, D. W., Olefsky, J. M., and Gustafson, T. A. Grb10 interacts differentially with the insulin receptor, insulin-like growth factor I receptor, and epidermal growth factor receptor via the Grb10 Src homology 2 (SH2) domain and a second novel domain located between the pleckstrin homology and SH2 domains. J Biol Chem 273, 6860-6867 (1998)
25. Kasus-Jacobi, A., Perdereau, D., Auzan, C., Clauser, E., Van Obberghen, E., Mauvais-Jarvis, F., Girard, J., and Burnol, A. F. Identification of the rat adapter Grb14 as an inhibitor of insulin actions. $J$ Biol Chem 273, 26026-26035 (1998)

26. Yaffe, M. B. Phosphotyrosine-binding domains in signal transduction. Nat Rev Mol Cell Biol 3, 177-186 (2002)

27. Pawson, T., Raina, M., and Nash, P. Interaction domains: from simple binding events to complex cellular behavior. FEBS Lett 513, 2-10 (2002)

28. Pawson, T., and Gish, G. D. SH2 and SH3 domains: from structure to function. Cell 71, 359-362 (1992)

29. Janes, P. W., Lackmann, M., Church, W. B., Sanderson, G. M., Sutherland, R. L., and Daly, R. J. Structural determinants of the interaction between the erbB2 receptor and the Src homology 2 domain of Grb7. J Biol Chem 272, 8490-8497 (1997)

30. Han, D. C., and Guan, J. L. Association of focal adhesion kinase with Grb7 and its role in cell migration. $J$ Biol Chem 274, 24425-24430 (1999)

31. Tanaka, S., Mori, M., Akiyoshi, T., Tanaka, Y., Mafune, K., Wands, J. R., and Sugimachi, K. A novel variant of human Grb7 is associated with invasive esophageal carcinoma. J Clin Invest 102, 821-827 (1998)

32. Pero, S. C., Oligino, L., Daly, R. J., Soden, A. L., Liu, C., Roller, P. P., Li, P., and Krag, D. N. Identification of novel non-phosphorylated ligands, which bind selectively to the SH2 domain of Grb7. J Biol Chem 277, 11918-11926 (2002)

33. Fiddes, R. J., Campbell, D. H., Janes, P. W., Sivertsen, S. P., Sasaki, H., Wallasch, C., and Daly, R. J. Analysis of Grb7 recruitment by heregulin-activated erbB receptors reveals a novel target selectivity for erbB3. J Biol Chem 273, 7717-7724 (1998)

34. Stein, D., Wu, J., Fuqua, S. A., Roonprapunt, C., Yajnik, V., D'Eustachio, P., Moskow, J. J., Buchberg, A. M., Osborne, C. K., and Margolis, B. The SH2 domain protein GRB-7 is co-amplified, overexpressed and in a tight complex with HER2 in breast cancer. Embo $J$ 13, 13311340 (1994)

35. Dong, L. Q., Du, H., Porter, S. G., Kolakowski, L. F., Jr., Lee, A. V., Mandarino, L. J., Fan, J., Yee, D., Liu, F., and Mandarino, J. Cloning, chromosome localization, expression, and characterization of an Src homology 2 and pleckstrin homology domain-containing insulin receptor binding protein hGrb10gamma. J Biol Chem 272, 2910429112 (1997)

36. Reilly, J. F., Mickey, G., and Maher, P. A. Association of fibroblast growth factor receptor 1 with the adaptor protein Grb14. Characterization of a new receptor binding partner. J Biol Chem 275, 7771-7778 (2000)

37. Cariou, B., Perdereau, D., Cailliau, K., Browaeys-Poly, E., Bereziat, V., Vasseur-Cognet, M., Girard, J., and Burnol, A. F. The adapter protein ZIP binds Grb14 and regulates its inhibitory action on insulin signaling by recruiting protein kinase Czeta. Mol Cell Biol 22, 69596970 (2002)

38. Jones, N., Master, Z., Jones, J., Bouchard, D., Gunji, Y., Sasaki, H., Daly, R., Alitalo, K., and Dumont, D. J. Identification of Tek/Tie2 binding partners. Binding to a 
multifunctional docking site mediates cell survival and migration. J Biol Chem 274, 30896-30905 (1999)

39. Almeida, E. A., Ilic, D., Han, Q., Hauck, C. R., Jin, F., Kawakatsu, H., Schlaepfer, D. D., and Damsky, C. H. Matrix survival signaling: from fibronectin via focal adhesion kinase to c-Jun NH(2)-terminal kinase. J Cell Biol 149, 741-754 (2000)

40. Tanaka, S., Sugimachi, K., Kawaguchi, H., Saeki, H., Ohno, S., and Wands, J. R. Grb7 signal transduction protein mediates metastatic progression of esophageal carcinoma. J Cell Physiol 183, 411-415 (2000)

41. Han, D. C., Shen, T. L., and Guan, J. L. Role of Grb7 targeting to focal contacts and its phosphorylation by focal adhesion kinase in regulation of cell migration. $J$ Biol Chem 275, 28911-28917 (2000)

42. Langlais, P., Dong, L. Q., Hu, D., and Liu, F. Identification of Grb10 as a direct substrate for members of the Src tyrosine kinase family. Oncogene 19, 2895-2903 (2000)

43. Giorgetti-Peraldi, S., Murdaca, J., Mas, J. C., and Van Obberghen, E. The adapter protein, Grb10, is a positive regulator of vascular endothelial growth factor signaling. Oncogene 20, 3959-3968 (2001)

44. Han, D. C., Shen, T. L., Miao, H., Wang, B., and Guan, J. L. EphB1 associates with Grb7 and regulates cell migration. J Biol Chem 277, 45655-45661 (2002)

45. Jahn, T., Seipel, P., Urschel, S., Peschel, C., and Duyster, J. Role for the adaptor protein Grb10 in the activation of Akt. Mol Cell Biol 22, 979-991 (2002)

46. Nantel, A., Huber, M., and Thomas, D. Y. Localization of endogenous Grb10 to the mitochondria and its interaction with the mitochondrial-associated Raf-1 pool. $J$ Biol Chem 274, 35719-35724 (1999)

47. Lemmon, M. A., and Schlessinger, J. Regulation of signal transduction and signal diversity by receptor oligomerization. Trends Biochem Sci 19, 459-463 (1994)

48. Angers, S., Salahpour, A., and Bouvier, M. Dimerization: an emerging concept for $\mathrm{G}$ protein-coupled receptor ontogeny and function. Annu Rev Pharmacol Toxicol 42, 409-435 (2002)

49. Brennan, P. J., Kumagai, T., Berezov, A., Murali, R., and Greene, M. I. HER2/Neu: mechanisms of dimerization/oligomerization. Oncogene 21, 328 (2002)

50. Aaronson, D. S., and Horvath, C. M. A road map for those who know JAK-STAT. Science 296, 1653-1655 (2002)

51. Dong, L. Q., Porter, S., Hu, D., and Liu, F. Inhibition of hGrb10 binding to the insulin receptor by functional domain-mediated oligomerization. J Biol Chem 273, 17720-17725 (1998)

52. Myers, M. G., Jr., Cheatham, B., Fisher, T. L., Jachna, B. R., Kahn, C. R., Backer, J. M., and White, M. F. Common and distinct elements in insulin and PDGF signaling. Ann N Y Acad Sci 766, 369-387 (1995)

53. Myers, M. G., Jr., Grammer, T. C., Brooks, J., Glasheen, E. M., Wang, L. M., Sun, X. J., Blenis, J., Pierce, J. H., and White, M. F. The pleckstrin homology domain in insulin receptor substrate-1 sensitizes insulin signaling. $J$ Biol Chem 270, 11715-11718 (1995)

54. Saltiel, A. R., and Pessin, J. E. Insulin signaling pathways in time and space. Trends Cell Biol 12, 65-71 (2002)
55. Morrione, A. Grb10 proteins in insulin-like growth factor and insulin receptor signaling (review). Int $J \mathrm{Mol}$ Med 5, 151-154 (2000)

56. Hansen, H., Svensson, U., Zhu, J., Laviola, L., Giorgino, F., Wolf, G., Smith, R. J., and Riedel, H. Interaction between the Grb10 SH2 domain and the insulin receptor carboxyl terminus. $J$ Biol Chem 271, 8882-8886 (1996)

57. Bereziat, V., Kasus-Jacobi, A., Perdereau, D., Cariou, B., Girard, J., and Burnol, A. F. Inhibition of insulin receptor catalytic activity by the molecular adapter Grb14. J Biol Chem 277, 4845-4852 (2002)

58. Stein, E. G., Gustafson, T. A., and Hubbard, S. R. The BPS domain of Grb10 inhibits the catalytic activity of the insulin and IGF1 receptors. FEBS Lett 493, 106-111 (2001) 59. Hemming, R., Agatep, R., Badiani, K., Wyant, K., Arthur, G., Gietz, R. D., and Triggs-Raine, B. Human growth factor receptor bound 14 binds the activated insulin receptor and alters the insulin-stimulated tyrosine phosphorylation levels of multiple proteins. Biochem Cell Biol 79, 21-32 (2001)

60. Wang, J., Dai, H., Yousaf, N., Moussaif, M., Deng, Y., Boufelliga, A., Swamy, O. R., Leone, M. E., and Riedel, H. Grb10, a positive, stimulatory signaling adapter in plateletderived growth factor BB-, insulin-like growth factor I-, and insulin-mediated mitogenesis. Mol Cell Biol 19, 62176228 (1999)

61. Morrione, A., Valentinis, B., Resnicoff, M., Xu, S., and Baserga, R. The role of mGrb10alpha in insulin-like growth factor I-mediated growth. $J$ Biol Chem 272, 26382-26387 (1997)

62. Wick, M. J., Dong, L. Q., Hu, D., Langlais, P., and Liu, F. Insulin receptor-mediated p62dok tyrosine phosphorylation at residues 362 and 398 plays distinct roles for binding GTPase-activating protein and Nck and is essential for inhibiting insulin-stimulated activation of Ras and Akt. J Biol Chem 276, 42843-42850 (2001)

63. Mounier, C., Lavoie, L., Dumas, V., Mohammad-Ali, K., Wu, J., Nantel, A., Bergeron, J. J., Thomas, D. Y., and Posner, B. I. Specific inhibition by hGRB10zeta of insulininduced glycogen synthase activation: evidence for a novel signaling pathway. Mol Cell Endocrinol 173, 15-27 (2001)

64. Guan, J. L. Role of focal adhesion kinase in integrin signaling. Int J Biochem Cell Biol 29, 1085-1096 (1997)

65. Schaller, M. D. Biochemical signals and biological responses elicited by the focal adhesion kinase.PG - 1-21. Biochim Biophys Acta 1540, 1-21 (2001)

66. Cary, L. A., and Guan, J. L. Focal adhesion kinase in integrin-mediated signaling. Front Biosci 4, D102-113 (1999)

67. Parsons, J. T., Martin, K. H., Slack, J. K., Taylor, J. M., and Weed, S. A. Focal adhesion kinase: a regulator of focal adhesion dynamics and cell movement.PG - 5606-13. Oncogene 19, 5606-5613 (2000)

68. Cary, L. A., Chang, J. F., and Guan, J. L. Stimulation of cell migration by overexpression of focal adhesion kinase and its association with Src and Fyn. J Cell Sci 109 ( Pt 7), 1787-1794 (1996)

69. Shen, T. L., and Guan, J. L. Differential regulation of cell migration and cell cycle progression by FAK complexes with Src, PI3K, Grb7 and Grb2 in focal contacts. FEBS Lett 499, 176-181 (2001) 
70. Reiske, H. R., Kao, S. C., Cary, L. A., Guan, J. L., Lai, J. F., and Chen, H. C. Requirement of phosphatidylinositol 3-kinase in focal adhesion kinase-promoted cell migration. J Biol Chem 274, 12361-12366 (1999)

71. Zhang, X., Chattopadhyay, A., Ji, Q. S., Owen, J. D., Ruest, P. J., Carpenter, G., and Hanks, S. K. Focal adhesion kinase promotes phospholipase C-gammal activity. Proc Natl Acad Sci U S A 96, 9021-9026 (1999)

72. Reiske, H. R., Zhao, J., Han, D. C., Cooper, L. A., and Guan, J. L. Analysis of FAK-associated signaling pathways in the regulation of cell cycle progression. FEBS Lett 486, 275-280 (2000)

73. Wilkinson, D. G. Eph receptors and ephrins: regulators of guidance and assembly. Int Rev Cytol 196, 177-244 (2000)

74. Adams, R. H., and Klein, R. Eph receptors and ephrin ligands. essential mediators of vascular development. Trends Cardiovasc Med 10, 183-188 (2000)

75. Stein, E., Cerretti, D. P., and Daniel, T. O. Ligand activation of ELK receptor tyrosine kinase promotes its association with Grb10 and Grb2 in vascular endothelial cells. J Biol Chem 271, 23588-23593 (1996)

76. Cary, L. A., Han, D. C., and Guan, J. L. Integrinmediated signal transduction pathways. Histol Histopathol 14, 1001-1009 (1999)

77. Lee, H., Volonte, D., Galbiati, F., Iyengar, P., Lublin, D. M., Bregman, D. B., Wilson, M. T., Campos-Gonzalez, R., Bouzahzah, B., Pestell, R. G., Scherer, P. E., and Lisanti, M. P. Constitutive and growth factor-regulated phosphorylation of caveolin-1 occurs at the same site (Tyr14) in vivo: identification of a c-Src/Cav-1/Grb7 signaling cassette. Mol Endocrinol 14, 1750-1775 (2000)

78. Samuel, D. S., Ewton, D. Z., Coolican, S. A., Petley, T. D., McWade, F. J., and Florini, J. R. Raf-1 activation stimulates proliferation and inhibits IGF-stimulated differentiation in L6A1 myoblasts. Horm Metab Res 31, 55-64 (1999)

79. Nantel, A., Mohammad-Ali, K., Sherk, J., Posner, B. I., and Thomas, D. Y. Interaction of the Grb10 adapter protein with the Raf1 and MEK1 kinases. J Biol Chem 273, 1047510484 (1998)

80. Manser, J., and Wood, W. B. Mutations affecting embryonic cell migrations in Caenorhabditis elegans. Dev Genet 11, 49-64 (1990)

81. Akiyama, S. K., Yamada, S. S., Yamada, K. M., and LaFlamme, S. E. Transmembrane signal transduction by integrin cytoplasmic domains expressed in single-subunit chimeras.PG - 15961-4. J Biol Chem 269, 15961-15964 (1994)

82. Tanaka, S., Mori, M., Akiyoshi, T., Tanaka, Y., Mafune, K., Wands, J. R., and Sugimachi, K. Coexpression of Grb7 with epidermal growth factor receptor or Her2/erbB2 in human advanced esophageal carcinoma. Cancer Res 57, 28-31 (1997)

83. Kauraniemi, P., Barlund, M., Monni, O., and Kallioniemi, A. New amplified and highly expressed genes discovered in the ERBB2 amplicon in breast cancer by cDNA microarrays. Cancer Res 61, 8235-8240 (2001)

84. Varis, A., Wolf, M., Monni, O., Vakkari, M. L., Kokkola, A., Moskaluk, C., Frierson, H., Jr., Powell, S. M., Knuutila, S., Kallioniemi, A., and El-Rifai, W. Targets of gene amplification and overexpression at $17 \mathrm{q}$ in gastric cancer. Cancer Res 62, 2625-2629 (2002)

85. Skotheim, R. I., Monni, O., Mousses, S., Fossa, S. D., Kallioniemi, O. P., Lothe, R. A., and Kallioniemi, A. New insights into testicular germ cell tumorigenesis from gene expression profiling. Cancer Res 62, 2359-2364 (2002)

86. Slamon, D. J., Godolphin, W., Jones, L. A., Holt, J. A., Wong, S. G., Keith, D. E., Levin, W. J., Stuart, S. G., Udove, J., Ullrich, A. Studies of the HER-2/neu protooncogene in human breast and ovarian cancer. Science 244, 707-712 (1989)

Abbreviations: Grb: growth factor receptor binding protein; FAK, focal adhesion kinase; FGF, fibroblast growth factor; EGF: epidermal growth factor; MAPK: mitogen activated kinase; $\mathrm{PKC}$, protein kinase $\mathrm{C}$; $\mathrm{PH}$, pleckstrin homology; RTK, receptor tyrosine kinase, $\mathrm{SH} 2$, Src-homology 2

Key words: Grb7, protein adaptors, phosphorylation, cell migration, cancer, mitogenesis, Review

Send correspondence to: Dr Jun-Lin Guan, Department of Molecular Medicine, Cornell University, Ithaca, New York 14853, Tel: 607-253-3586; Fax: 607-253-3708; E-mail : jg19@cornell.edu 